\title{
Performance of Measles Case-based Surveillance System in Rivers State, Nigeria 2011-2018
}

\author{
Rowland Utulu' ${ }^{1 *}$, Joseph Urang ${ }^{3}$, Aishat Usman ${ }^{1,2}$, Neni Aworabhi ${ }^{1}$, Ugochukwu Osigwe ${ }^{2}$, Muhammad Shakir \\ Balogun ${ }^{1,2}$, Eniola A. Bamgboye ${ }^{4}$ \\ 'Nigeria Field Epidemiology and Laboratory Training Program \\ ${ }^{2}$ African Field Epidemiology Network (AFENET), Abuja \\ ${ }^{3}$ Rivers State Primary Healthcare Management Board, Port-Harcourt, Rivers state, Nigeria \\ ${ }^{4}$ Department of epidemiology and medical statistics, faculty of public health, University of Ibadan, Nigeria
}

\section{Article Info}

\section{Article Notes}

Received: February 29, 2020

Accepted: March 30, 2020

\section{*Correspondence:}

Dr. Rowland Utulu, Community Medicine Department, Alex Ekwueme Federal University Teaching Hospital Abakalik (AEFUTHA), Ebonyi State, Nigeria; Field Epidemiology Resident, Nigeria Field Epidemiology and Laboratory Training Program, Nigeria; Telephone No: +234(0) 8065662895; Email: rhowly1@gmail.com.

C 2020 Utulu R. This article is distributed under the terms of the Creative Commons Attribution 4.0 International License.

\section{Keywords:}

Case-based surveillance

Measles

Performance

Surveillance indicators

Rivers State

Nigeria

\section{ABSTRACT}

Background: WHO African region set a target for elimination of measles by 2020 and recommended member states adopt a case-based surveillance system. WHO AFRO guidelines for measles surveillance state that an optimally performing surveillance system is crucial to elimination of measles. Rivers State is one of the high burden states for measles in southern Nigeria.

Objective: This study assessed the performance of the measles case-based surveillance in Rivers state, Nigeria.

Methods: We reviewed measles case-based surveillance data in Rivers state, Nigeria from year 2011-2018.

Results: A total of 1,731 suspected cases were reported with 1,128 (65.2\%) confirmed cases of measles. Majority were confirmed by epidemiologic linkage 907 (80.4\%) while laboratory confirmed cases constituted 206 (18.3\%). Age group 1-4 years was the most affected 443 (42.6\%). For cases with vaccination status available 425 (53.1\%) were unvaccinated. No discarded cases were reported from 2016 to 2018. Non-measles febrile rash illness rate target of $\geq 2$ per 100,000 was not achieved at any point in the eight-year period. The proportion of districts/LGAs reporting $>2$ Non Measles-Non Rubella Febrile Rash Illness cases/year was above the minimum target of $80 \%$ in just four years.

Conclusion: The surveillance system performed poorly with sensitivity and representativeness less than optimal. Measles elimination must leverage upon existing structures for polio elimination to improve surveillance. A more detailed analysis of the system is essential to identify all the gaps that may retard elimination efforts.

\section{Introduction}

Measles elimination is defined as the absence of endemic measles virus transmission in a defined geographic area for at least 12 months in the presence of a well-performing surveillance system ${ }^{1}$. The World Health Organization recommends that as countries prepare for elimination, surveillance for measles be active and case-based with laboratory investigation of cases rather than passive reporting of cases in pre-elimination era ${ }^{2}$. Case-based surveillance system for measles entails investigation of every case with blood samples taken for laboratory confirmation of measles-specific antibody (IgM).

In 2011, the WHO African region set a goal which was to achieve elimination of measles by the year $2020^{1}$. The three main strategies to be employed were achieving high routine immunization, high supplemental immunization activities coverage 
(SIAs) and case-based surveillance system with improved case management. Four targets that were crucial for elimination were set; 1) 95\% coverage with first dose of measles vaccine at the national level and at every district/ local government area (LGA) 2) 95\% coverage with SIAs in every district 3) Measles incidence of $<1$ per million population 4) Reaching targets for two of the main surveillance performance indicators. These indicators monitor key aspects of the surveillance system and ensure timely actions. The two main surveillance performance indicators are 1) Non-measles, non-rubella febrile rash illness rate ( $>2 / 100,000$ population) and 2) Proportion of districts $(80 \%)$ that have reported at least 2 suspected case of measles with a blood specimen per year. The main indicators assess the sensitivity of the surveillance system and the extent to which cases identified during active search represent all cases within the entire districts under surveillance. These are key attributes that assess the strength of the surveillance system and need to be above the stated minimum requirements to achieve elimination.

Studies seem to suggest minimal progress in the African region in achieving elimination targets compared to other regions ${ }^{3}$. Efforts towards elimination of measles in Nigeria has steadily ramped up, given the recent achievements in polio eradication. However, the performance of measles case-based surveillance system in the different states of the country is still unclear and has not been properly documented. Rivers State is one of the most populated states in the country and the most populated in the Southsouth geopolitical zone of Nigeria and reports indicate frequent outbreaks of measles in Rivers State. Some of these outbreaks are reported to the national level while others remain unreported. Parts of northern Nigeria have received significant attention due to the security challenges that have made some areas inaccessible and retarded disease elimination efforts. The riverine and marshy terrain of Rivers State also poses some challenge to elimination efforts. Located in the oil rich region of the Niger-delta, it is plagued by frequent unrest not unlike parts of the northern region, due to activities of several militant factions active in the region ${ }^{4-6}$. Abductions and robberies are a frequent occurrence, consequently some health centers have been abandoned with the settlements within that catchment area underserved and children not being appropriately immunized for age ${ }^{4-6}$. Nigeria has currently revised its elimination target to 2028 in light of the unlikely chance of accomplishing the 2020 target. Consequently, it is essential to establish current performance of the measles casebased surveillance system, highlight the gaps that exist and what improvement may be needed to achieve elimination. We therefore assessed the performance of the Rivers State measles case-based surveillance system and monitor its progress towards elimination.

\section{Methods}

\section{Study setting}

Rivers state has an estimated projected population of $7,809,035$ million using a population growth rate of 3.4 in $2018^{7,8}$. It is located in the South-south geopolitical zone of the country and is the $6^{\text {th }}$ largest state in Nigeria9,10. It has 23 local government areas (LGA) and the largest being the capital city of Port-Harcourt LGA ${ }^{9,10}$.

\section{Study design}

We conducted a secondary analysis of measles/rubella case-based surveillance data for Rivers State for the period of January 2011 to December 2018.

\section{Study population/Data source}

We obtained measles/rubella case-based data from 2011-2018 from the Rivers State Primary Healthcare Management Board and WHO field office in Port-Harcourt.

\section{Case definitions}

Suspected case: A suspected case was defined as the occurrence of fever and a maculopapular rash with any one of cough, coryza, or conjunctivitis or any illness in a person that a clinician suspects to be measles ${ }^{1}$.

Confirmed case: A confirmed measles case is classified as either laboratory-confirmed, epidemiologically linked, or clinically compatible ${ }^{1}$.

- Laboratory confirmed case: Laboratory confirmation is when measles-specific IgM antibody is detected in serum by enzyme-linked immunosorbent assay (ELISA) in the absence of measles vaccination within 30 days before specimen collection ${ }^{1}$.

- Epidemiological linkage: A suspect measles case is said to be epidemiologically linked to a confirmed case when the patient has had contact with or lives in the same locality as a person with laboratory-confirmed measles infection. ${ }^{1}$

- Clinically compatible: These are those cases which satisfy the clinical case definition, but a laboratory test or an epidemiological link is lacking ${ }^{1}$.

\section{Measles surveillance system characteristics}

When a case of measles is notified, a case investigation form is completed with the appropriate demographic and clinical features recorded. A blood sample is taken for assessment of measles IgM by Enzyme Linked Immunosorbent Assay (ELISA). The most ideal time for sample collection being between 4-28 days after the rash onset. Samples from Rivers State are sent to the Central Public Health Laboratory in Yaba, Lagos which is the closest 


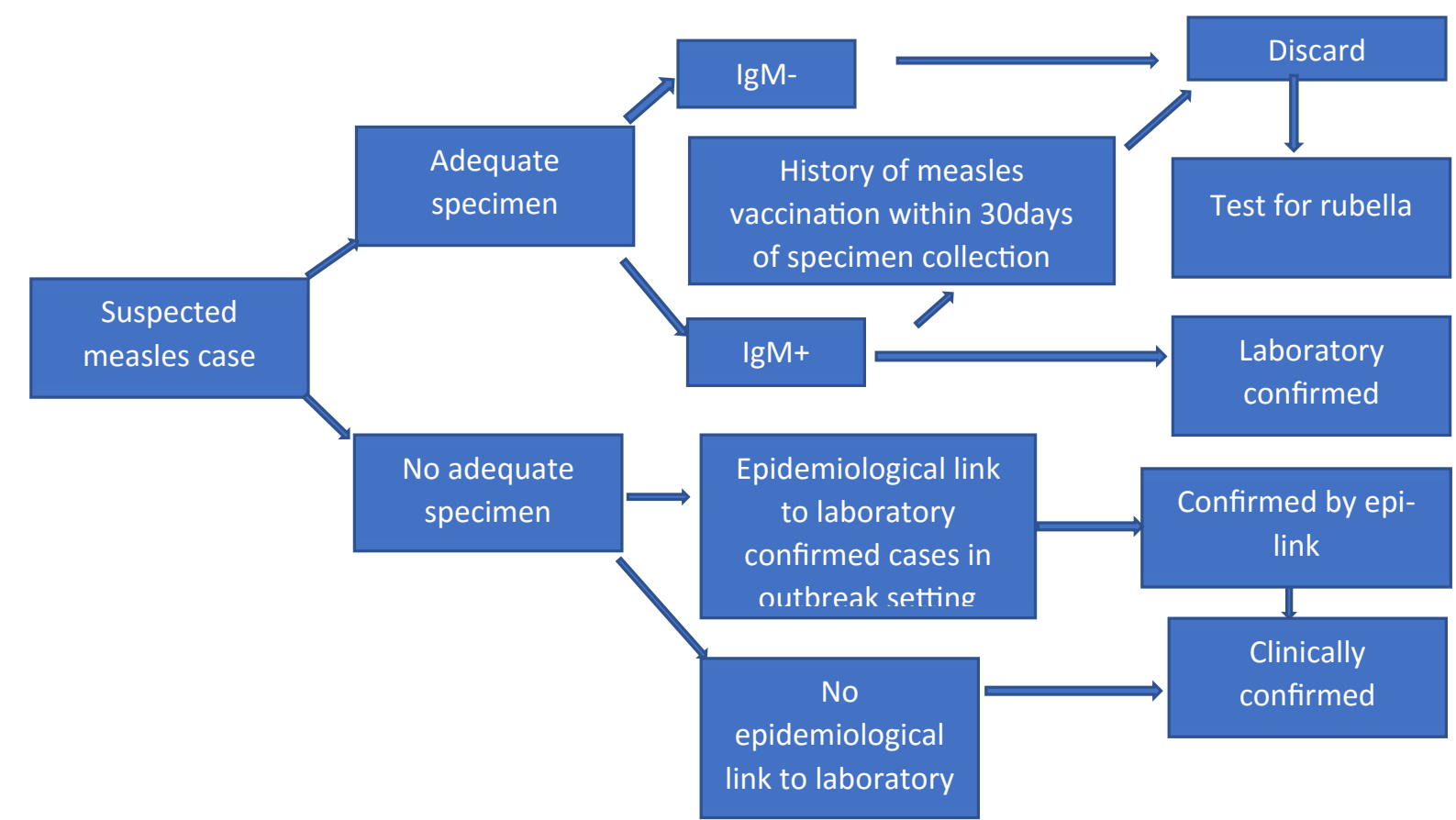

Image adopted from the African regional guidelines for Measles and Rubella surveillance ${ }^{1}$

Figure 1: Measles case classification algorithm

of the four reference laboratories for measles diagnosis in the country and feedback sent after results are available.

\section{Assessment of surveillance performance}

Surveillance performance was assessed using standard indicators recommended in the WHO African regional guidelines for measles and rubella surveillance ${ }^{1}$. Three principal and four supplementary indicators were estimated. The indicators assessed were

Non-measles febrile rash illness rate per $100,000^{1}$.

Proportion of LGAs/districts reporting > 2 Non-Measles febrile rash illness cases/year ${ }^{1}$.

Proportion of LGAs that have reported at least 1 case of measles with a blood specimen per year ${ }^{1}$.

Annualized rate of investigation (with blood specimen) per 100,000 per year ${ }^{1}$.

Proportion of specimen arriving the laboratory within 3days (this was defined as time from specimen collection to arrival at the laboratory).

Proportion of specimen reaching the laboratory in good condition ${ }^{1}$.

Timeliness of case investigation (time from date of onset of disease to case investigation with specimen collection) within 3days.

Frequencies and proportions were estimated for each of these indicators and were compared to the WHO standard values. The method used in the estimation of the indicators is stated in the referenced document. ${ }^{1}$

\section{Data analysis and management}

Two excel sheets containing data from 2011-2018 were used for the analysis. Prior to analysis, columns with similar variables such as age, sex were merged in a separate excel workbook before analysis. One reporting template was used from 2011-2016 which contained more variables than the other data template which contained data from 2017-2018 with considerably less information. The first reporting template contained information on the date the cases were seen at the health facility, date when the samples were received at the national level, date when the district/LGAs got feedback and vaccination status but were absent on the second reporting template from 2017 to 2018 . Variables with over $30 \%$ of data unavailable were noted but excluded from analysis. Data was cleaned on Microsoft Excel and preliminary analysis was conducted before importing to Epi Info 7.2 for further analysis. We calculated frequencies and proportions for variables such as age, sex, number of vaccine doses, specimen quality, LGA of residence, measles IgM test result and other relevant variables. Indicators were calculated and compared with the set standard. Data was protected using a password and information was stored on one laptop and on google drive that was only accessible to the research team.

\section{Ethics}

Ethical waiver was provided by the ethical review board 
of the Rivers State ministry of health with the ethical review number MH/PRS/391/VOL.2/617. A full ethical review was not needed as it was a secondary data analysis and data was de-identified to ensure confidentiality. The State Immunization Officer for Rivers State Primary Healthcare Management Board (RSPHCMB) granted permission for this study.

\section{Results}

The analysis identified several missing data fields on both data templates. Information on sex and LGA of residence was complete. Variables such as specimen condition, measles IgM status had very little missing data (2\%). However, date of birth was missing for $7 \%$ of cases, $13 \%$ on the date cases were seen at the health facility, $14 \%$ on the date specimen was sent to and received at the laboratory, $27 \%$ on the vaccination status of cases, $57 \%$ on the date of last vaccination of cases. Some of the variables were absent from the second data template used from 2017 to 2018.

There were 1,731 suspected measles cases reported from January 2011 to December 2018 in Rivers State, out of which 1,128 (65.2\%) were confirmed while 227 (13.1\%) were discarded.

Table 1 shows that 606 (53.8\%) confirmed cases were males and age group 1-4 years accounted for the highest number among the confirmed cases with 443 (42.6\%) cases. Vaccination status of cases was only available from 2011-2015 with 425 (53.1\%) out of 801 confirmed cases

Table 1: Characteristics of measles cases in Rivers state 2011-2018

\begin{tabular}{|l|c|c|c|}
\hline Variables & $\begin{array}{c}\text { Suspected } \\
\text { cases n (\%) }\end{array}$ & $\begin{array}{c}\text { Confirmed } \\
\text { cases n (\%) }\end{array}$ & \\
\hline *Sex & & $\begin{array}{c}\text { \#Sex-specific Incidence } \\
\text { rate per 100,000 }\end{array}$ \\
\hline Male & $902(52)$ & $606(53.8)$ & 16.8 \\
\hline Female & $828(48)$ & $521(46.3)$ & 15.1 \\
\hline *Age group & & & $\begin{array}{c}\text { Age-specific incidence } \\
\text { rate per 100,000 }\end{array}$ \\
\hline$<1$ & $286(18)$ & $184(17.7)$ & 65.7 \\
\hline $1-4$ & $644(40.6)$ & $443(42.6)$ & 39.5 \\
\hline $5-14$ & $427(26.9)$ & $272(26.2)$ & 14.1 \\
\hline$\geq 15$ & $229(14.4)$ & $141(13.6)$ & 3.8 \\
\hline Vaccination & & & \\
\hline status** & & & \\
\hline 0 & $673(53.2)$ & $425(53.1)$ & \\
\hline 1 & $458(36.2)$ & $290(36.2)$ & \\
\hline 2 & $134(10.6)$ & $86(10.7)$ & \\
\hline
\end{tabular}

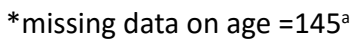

$*$ missing data on sex $=1^{\mathrm{b}}$

${ }^{* *}$ missing data on vaccination status $=465^{c}$

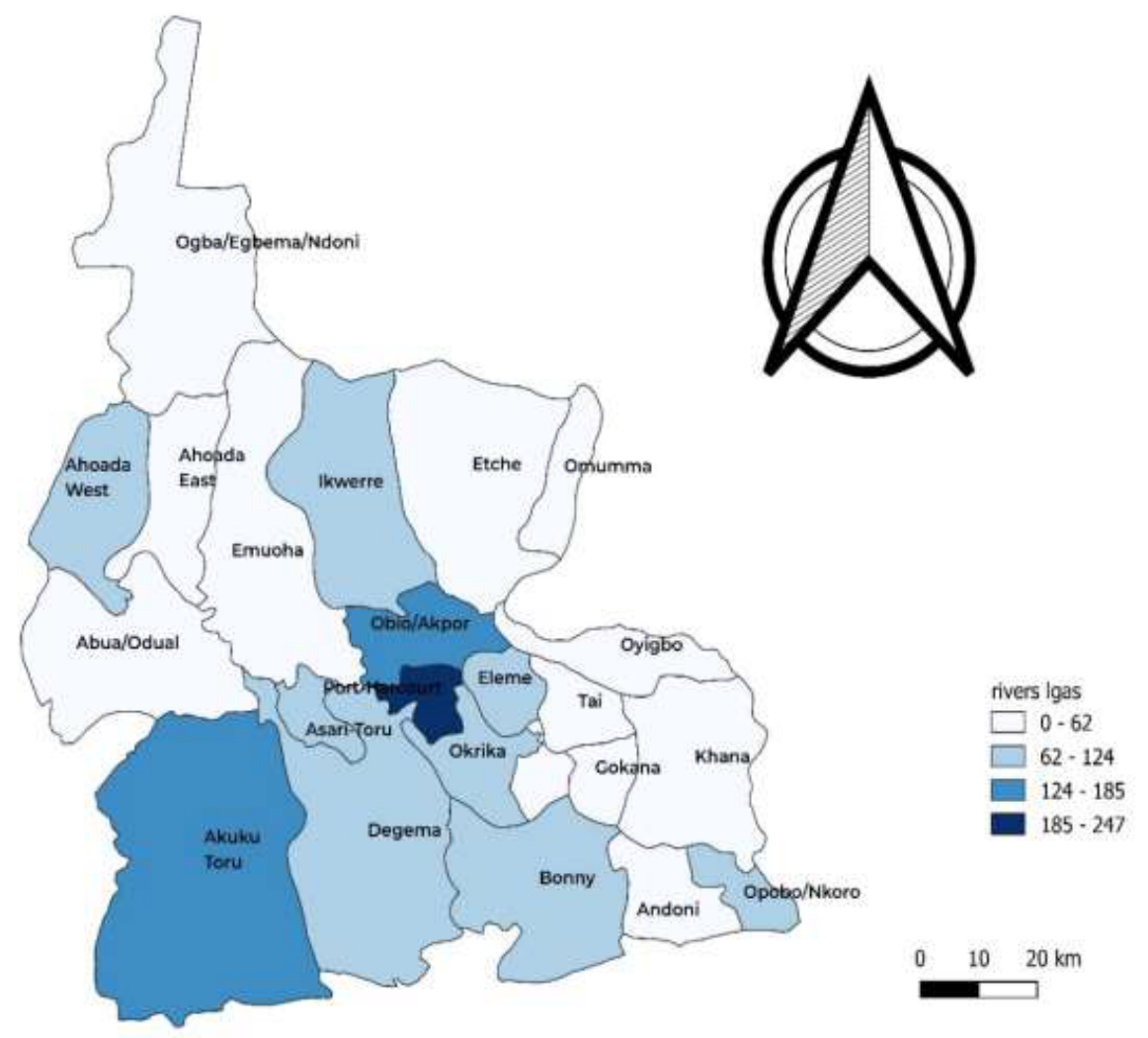

Figure 2: Map of Rivers state showing the distribution of suspected measles cases

The map in figure 2 shows the distribution of suspected cases during the period under study with Port-Harcourt LGA having with the largest proportion of cases 247 (14.3\%), and Ogu Bolo with the lowest number of cases reported 19 (1.6\%) 


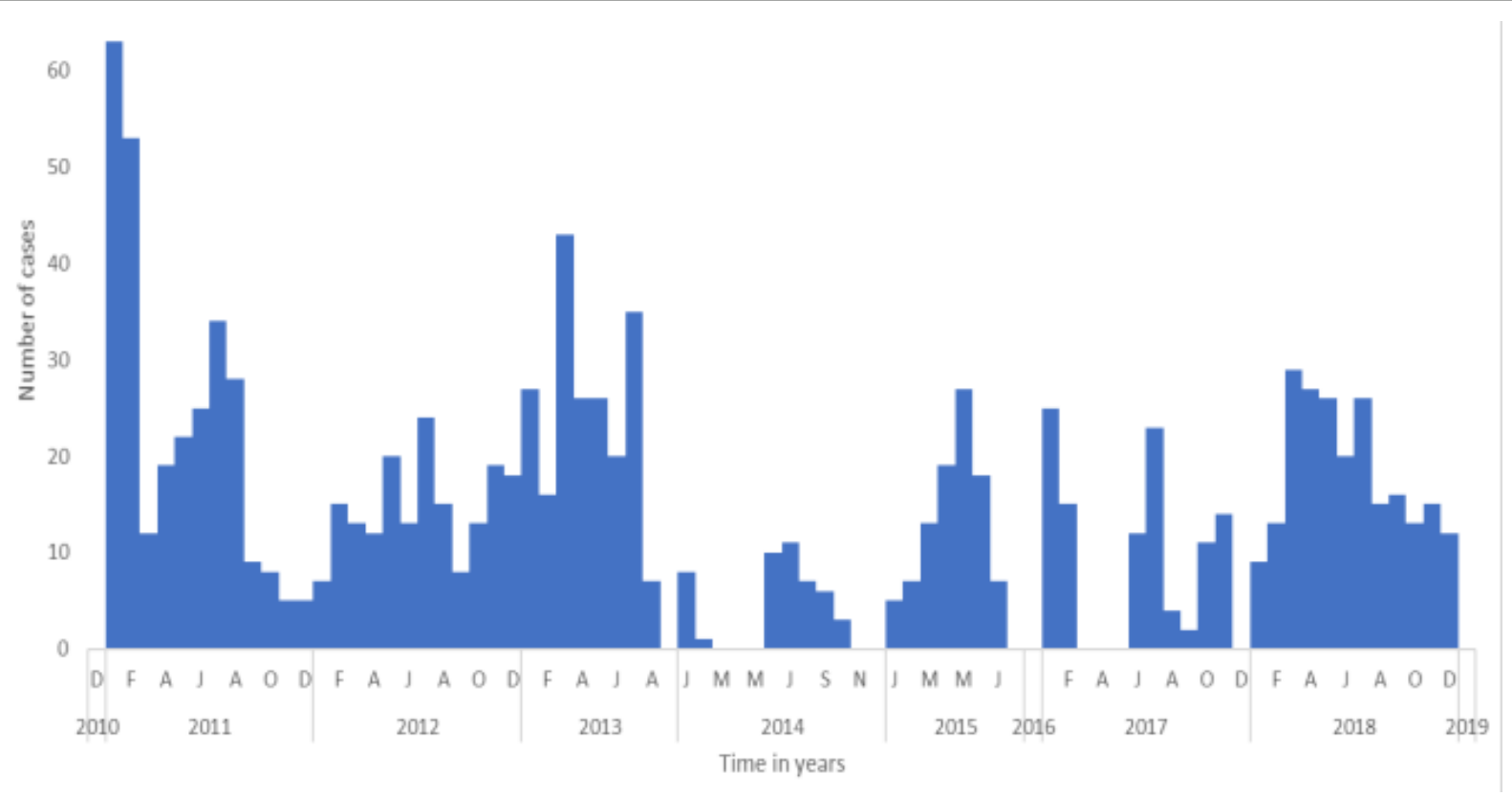

Figure 3: Epidemic curve showing the monthly occurrence of confirmed measles cases

Figure 3 shows the seasonal occurrence of confirmed cases of measles in Rivers State. The highest peak was in January 2011, with a sharp decline in cases in the following months with another peak in July of that same year. In almost all the years from 2011-2018, there was a rise in reported cases from March to June of each year except in 2014, 2016 and 2017 (no confirmed cases in 2016). January 2018 was marked by a gradual rise in the number of confirmed cases reported till June, then levels slowly declined.

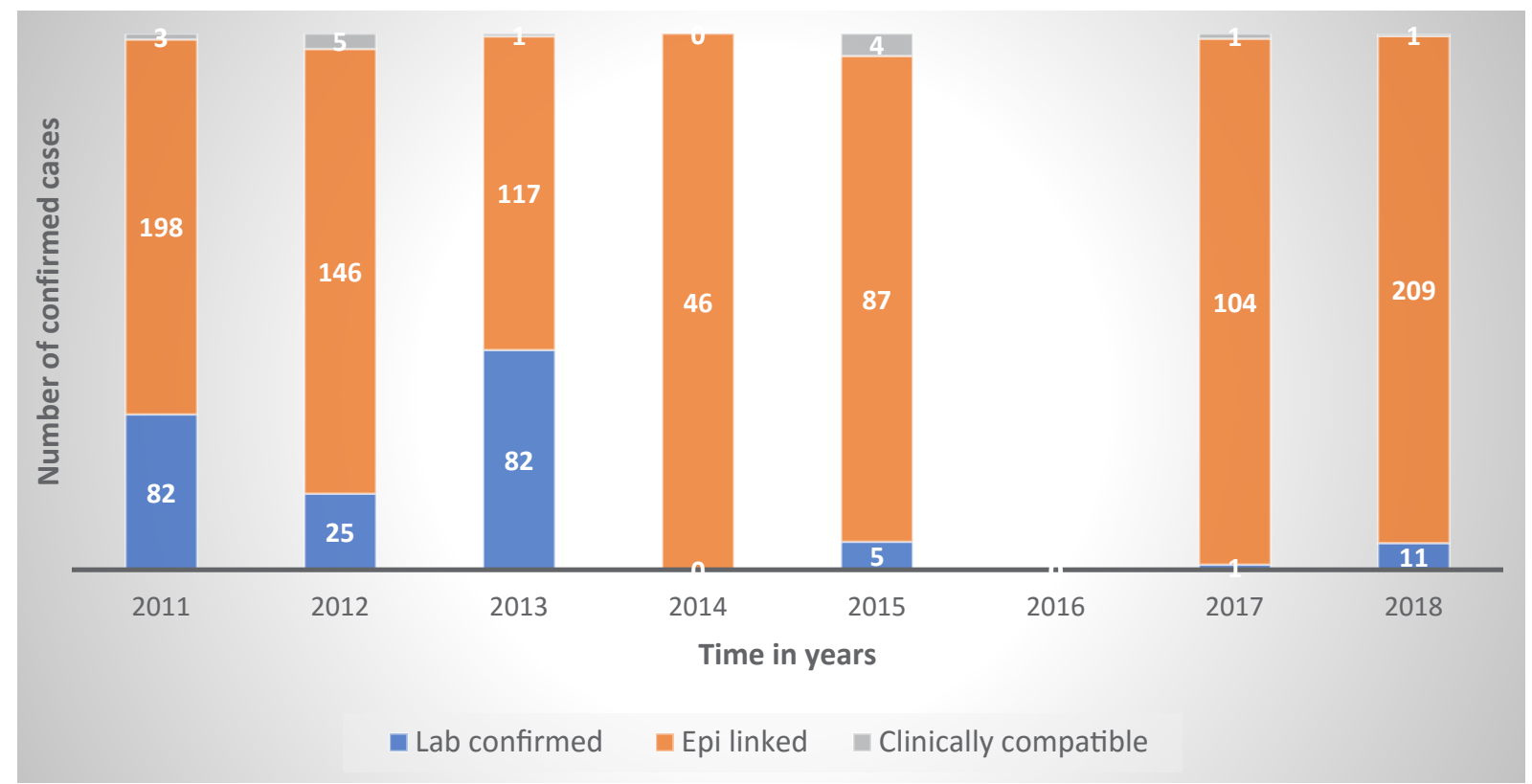

Figure 4: Proportion of confirmed measles cases in Rivers state from 2011-2018

Figure 4 shows the distribution of confirmed cases reported during the period. The majority of cases were confirmed epidemiologically [907 (80.4\%)] and clinically confirmed accounted for the smallest number [15 (1.3\%)]. Laboratory confirmation of cases declined over time with no laboratory confirmed cases in 2014, 2016 and only one case in 2017.

in this period being unvaccinated, while 376 (46.9\%) had at least one dose of the measles vaccine.

The main measles surveillance performance indicators such as non-measles febrile rash illness rate target of $\geq 2$ per 100,000 was not achieved throughout the entire period under review (Table 2). The proportion of LGAs reporting $>2$ non-measles non-rubella febrile rash illness cases/year was above the minimum target of $80 \%$ for only three years (2011-2014). The target for the proportion of LGAs that reported at least 1 case of measles with a blood specimen 
Table 2: Measles surveillance performance indicators

\begin{tabular}{|c|c|c|c|c|c|c|c|c|c|c|c|c|}
\hline & \multirow[b]{2}{*}{ Available Measles surveillance indicators } & \multirow[b]{2}{*}{ Target } & \multicolumn{10}{|c|}{ Year under surveillance } \\
\hline & & & 2011 & 2012 & 2013 & 2014 & 2015 & 2016 & 2017 & 2018 & Mean result & $\begin{array}{l}\text { Years target } \\
\text { was met }\end{array}$ \\
\hline & Suspected measles cases & & 294 & 193 & 313 & 144 & 222 & 75 & 212 & 254 & 214 & NA \\
\hline 1. & $\begin{array}{l}\text { *Non-measles febrile rash illness rate } \\
\text { per } 100,000\end{array}$ & $\geq 2$ & 0 & 0.27 & 1.8 & 1.5 & 0 & 0 & 0 & 0 & 1.2 & 0 \\
\hline 2. & $\begin{array}{l}\text { *Proportion of LGAs reporting }>2 \\
\text { Non-Measles febrile rash illness cases/ } \\
\text { year }\end{array}$ & $\geq 80 \%$ & 100 & 100 & 100 & 100 & 65 & $\#$ & $\#$ & $\#$ & 93 & 4 \\
\hline 3. & $\begin{array}{l}\text { *Proportion of LGAs that have reported } \\
\text { at least } 1 \text { case of measles with a blood } \\
\text { specimen per year }\end{array}$ & $\geq 80 \%$ & 100 & 100 & 100 & 73.9 & 73.9 & $\#$ & 100 & 100 & 92.5 & 6 \\
\hline 4. & $\begin{array}{l}\text { Annualized rate of investigation per } \\
100,000 \text { per year }\end{array}$ & $>1$ & 4.9 & 3.1 & 4.9 & 2.2 & 3.3 & 1.1 & 3.0 & 3.5 & 2.6 & 8 \\
\hline 5. & $\begin{array}{l}\text { Proportion of specimen arriving the lab } \\
\text { within 3days }\end{array}$ & $\geq 80 \%$ & 47 & 60 & 60 & 61 & 54 & 80 & 37 & 32 & 54 & 1 \\
\hline 6. & $\begin{array}{l}\text { Proportion of specimen reaching the } \\
\text { lab in good condition }\end{array}$ & $\geq 90 \%$ & 100 & 96 & 96 & 99 & 100 & 100 & 100 & 99 & 98.8 & 8 \\
\hline 7. & $\begin{array}{l}\text { Timeliness of case investigation within } \\
\text { 3days }\end{array}$ & $\geq 80 \%$ & 33 & 27 & 33 & 33 & 41 & 53 & 38 & 43 & 38 & 0 \\
\hline
\end{tabular}

*Main measles performance indicators

\#Information not available, hence could not be estimated

NA (not applicable)

per year was achieved in every year but from 2014-2016. Annualized rate of investigation (with blood specimens) of suspected measles/rubella cases was above the target of $>1$ case per 100,000 population per year throughout the eight-year period. Proportion of blood specimens arriving at laboratory within 3 days of being taken (target $>80 \%$ ) was consistently less than target except in 2016. However, in 2016, data was only available on five specimen samples sent to the laboratory.

The proportion of investigated cases that were confirmed by serologic testing to be measles was averagely $12.7 \%$ (206) over the entire period of 2011-2018 and above the WHO-recommended target of $10 \%$. The highest proportion of investigated cases confirmed serologically to be measles was in 2011 with $28 \%$, and none were confirmed to be measles by serology in 2014 and 2016 .

The proportion of specimen reaching the laboratory in good condition was consistently above the WHOrecommended target of $90 \%$ across the entire period under study.

\section{Discussion}

This study assessed the performance of the measles case-based surveillance system in Rivers State by analyzing data that was collected on two separate templates. The reason for this change in the reporting template was unclear but the second data template contained data from other states in the south-south region of the country that used this particular template. This change of the formerly used reporting template may have been an attempt at uniformity with other states in the region without serious consideration of the implications. The first template provides more information relevant for better monitoring and assessment of the surveillance system.

Missing data and inconsistencies in the available data prevented a more robust assessment of the surveillance system and limits some of what can be inferred from this data. Several variables needed to assess the surveillance system were inadequate and this was a recurrent problem on both data templates. The vaccination status of cases is crucial to understand the reason behind continued transmission of the disease. It can provide information about access to the immunization, the cold chain system, the effectiveness of the vaccine among others. But this information was present on only the first data template. The absence of vaccination information is similar to other studies in the African region ${ }^{11,12}$. Results of some samples collected were still pending even after one year and this must be understood in interpretation of the information from this study, as it could mean there were more cases in that year than this study reports. In 2016, there were no confirmed cases due to reagents being damaged, preventing samples from being run, so every case in 2016 is reported as pending. This suggests quality assurance issues in the laboratory in addition to the shortfalls in reporting practices. The reagents damaged that year were not replaced as quickly due to funding issues that year. Reports from the laboratory detail improved funding in the succeeding years after 2016 and consequently, a non-reoccurrence of the problem. Furthermore, with the successes achieved in polio eradication in the country, as 
it enters the precertification phase, improved funding for surveillance activities regarding measles.

There were no significant sex differences among the confirmed cases. In absolute numbers, the age distribution of cases seen in this study saw 1-4 years (41.4\%) accounting for the most number of cases and those over the age of 15 years $(14.8 \%)$ being the least affected was comparable to the age distribution of cases seen in studies in other African countries ${ }^{11,12}$. However, the age-specific incidence rate when calculated shows a higher risk among children under the age of 1 year in the general population and the lowest risk was in those older than 15 years and above. This information underscores the need for improving the timeliness of immunization rather than just completeness of immunization.

The surveillance system was subpar for most of the indicators used in assessing its performance. This was a similar finding with a study carried out to assess regional efforts towards measles elimination in Africa that reported little to no progress in the performance of the measles casebased surveillance system ${ }^{3}$. The main indicators assess sensitivity and representativeness of the surveillance system. The indicator for sensitivity of the measles casebased surveillance system is the non-febrile measles rash illness rate per $100,000^{2}$. Sensitivity never met the recommended target throughout the entire period and seemed to decline even further from 2015 . This was vastly different from other studies in Nepal and another study carried out in South-West geopolitical zone of Nigeria that showed steady progress with sensitivity increasing over time and another study in Kenya that met the target in all years under review ${ }^{11,13,14}$. The decline in 2015 was in part due to a 6-month strike action by health workers at the LGA level leading to a failure to report cases. This was the longest strike action during the period under study, although there were several short strike actions by health workers. Representativeness, which was measured by the proportion of LGAs reporting $>2$ non-measles febrile rash illness cases/year, showed some improvement in the early years but declined in the succeeding years of the study ${ }^{2}$. The results obtained in this study for representativeness were much lower than another study in the country but similar to a national study of Nepal and Kenya ${ }^{11,13,14}$. The target for representativeness was met for four out of the eight years and declined after 2016. Sensitivity and representativeness of the surveillance system were poor due to the use of discarded cases in the computation of these indices, none of which was reported from 2016. This lack of discarded cases was likely due to quality issues at the laboratory rather than a major reporting issue because the number of suspected cases reported remained fairly high, but a larger proportion was classified as pending. In the five years from 2011 to 2015 there were 171 pending cases compared to 215 in just the three years from 2016 to 2018. In 2016, reports state that reagents for processing measles samples were damaged and so samples were not processed. These two indicators are the main indicators used to assess performance of the measles case-based surveillance system and the results seemingly indicate a poorly performing system.

However, some of the supplementary indicators such as the annualized rates of investigation and proportion of LGAs that reported at least 1 case of measles with a blood specimen per year showed some progress, with the former meeting the target in every year and the latter meeting the target in at least 6 of the 8 years under study. This was similar to a study in western Nigeria where annualized rates of investigation were above the recommended target but dissimilar to another study in Zimbabwe that recorded a decline ${ }^{12,13}$. The aforementioned are considered supplementary indicators but also provide further information on the sensitivity and representativeness of the surveillance. These showed some progress and were not as poor as the main indicators which depend on the proportion of discarded cases. This further suggests laboratory quality assurance issues and the need for further investigation. Commendable aspects of the surveillance system were the proportion of specimen reaching the laboratory in good condition which was very high throughout the entire period of the study.

As Nigeria enters the polio free certification phase, the attention is rapidly turning to measles and it is expected that this reporting of cases and the speed of investigation will improve. The proportion of specimen reaching the laboratory in less than three days never met the target throughout the period of the study. This is largely due to the paucity of reference laboratories capable of diagnosing measles in the country with the closest to the capital city of Port Harcourt being 10 hours away. Consequently, samples were pooled before being transported to the reference laboratory in Lagos, Nigeria.

The number of reported cases remained relatively high with just under 100 reported cases in one year during the entire period under review. These results show ongoing transmission of measles with a mild dip in the middle years and then the incidence began to rise again till a peak in 2018.

Some other reasons for the poor performance of the surveillance system may be the difficulty experienced by health workers in reaching some areas for two major reasons. Firstly, the terrain of Rivers State is in the rainforest region with several areas inaccessible by road and hidden by dense forest vegetation. Secondly, Rivers State is a key oil producing state in the Niger Delta region and consequently several militant and criminal groups are present in parts of the state. There have been abductions and attacks on 
primary healthcare workers limiting immunization efforts and outbreak response activities. These have served to maintain the high incidence of measles seen in the state.

Surveys such the National Demographic and Health Survey (NDHS) 2013 \& 2018 reported over 70\% immunization coverage for measles containing vaccine for the first dose (MCV 1) in children aged 12-23 months in the state ${ }^{15,16}$. Other surveys like the National Nutrition and Health Surveys of 2015 \& 2018 reported rates between $40-61 \%$ for children aged $12-23 m_{0}$ ths $^{16,17}$. Nigeria recently introduced MCV 2 and is currently been piloted in several states in the country including Rivers. Measles elimination requires at least 95\% coverage with MCV 2 administered at 15 months to achieve herd immunity and prevent outbreaks ${ }^{18}$. The reality of the inability to meet the WHO AFRO target of measles elimination by 2020 was the reason behind the new target for measles elimination in Nigeria set for 2028. In order to achieve the new measles elimination target of 2028 in Nigeria then two key aspects must improve. Routine immunization coverage must improve as well as over 95\% coverage with supplemental immunization activities (SIAs). The surveillance system will need strengthening, particularly by leveraging heavily upon existing infrastructure for active surveillance of polio. Key attention needs to be paid to quality control in the reference laboratory with frequent quality assurance measures being taken and strict guidelines to improved turnaround time for sample processing. Proper monitoring to ensure that results are updated on the line list immediately these results are available.

The findings of this study, must however be interpreted in light of the limitations in the quality of data. The lack of information on the measles IgM status in some cases, pending status of others and particularly in 2016 with all cases not being properly analyzed by the laboratory significantly impacts on the conclusion to be drawn from this study. It could mean that there was an actual reduction in confirmed cases over time or a higher incidence of measles in the studied population than is reported by this study.

\section{Conclusion}

This analysis revealed a surveillance system performing below WHO recommended standards. It also highlighted data management and possible laboratory quality control issues. The high incidence of measles in this study combined with the flailing surveillance system bode poorly for measles elimination in the state. However, these issues may have been recognized and will hopefully be a focus for the new program for elimination in the country.

\section{Acknowledgement}

We would like to thank WHO Rivers State field office for their assistance with data for this project, Dr. Wari Tamuno Numbere, Dr. Roland Obed-Whyte and Rivers State Primary Healthcare Management Board, Rivers state, Nigeria for their support for this project. This study was privately funded by the authors.

\section{Conflict of Interest}

$$
\text { None }
$$

\section{Funding}

None

\section{Author Contributions}

\section{Conception - Rowland Utulu}

2. Acquisition, analysis and interpretation of data for the work - Rowland Utulu, Joseph Urang, Neni Aworabhi, Ugochukwu Osigwe, Eniola A. Bamgboye

3. Drafting and revising the work - Rowland Utulu, Muhammad Shakir Balogun, Aishat Usman, Ugochukwu Osigwe

4. Final approval of the work to be published - Rowland Utulu, Joseph Urang, Neni Aworabhi, Muhammad Shakir Balogun, Aishat Usman, Ugochukwu Osigwe, Eniola A.Bamgboye

\section{References}

1. Office WHOR. AFRICAN REGIONAL GUIDELINES FOR MEASLES AND RUBELLA SURVEILLANCE WHO Regional Office for Africa. 2015;(April):1-82.

2. Organization WH. Measles - Vaccine-Preventable Diseases Surveillance standards. WwwImmunizeCa [Internet]. 2011;1-30. Available from: http://www.phac-aspc.gc.ca/im/vpd-mev/measlesrougeole-eng.php

3. Masresha B, Katsande R, Luce R, et al. Performance of National Measles Case-Based Surveillance Systems in The WHO African Region 2012 - 2016. J Immunol Sci. 2018; 2(SI1): 130-4.

4. Kpae G. Mobile Communication Unemployment and Armed Robbery in Port Harcourt.

5. Edozie V. Rivers State under criminal attacks. Daily Trust [Internet]. 2016 Mar 10 [cited 2020 Jan 9]; Available from: https://www. dailytrust.com.ng/news/crime/rivers-state-under-criminalattacks/137237.html

6. Crime in Rivers State - Wikipedia [Internet]. [cited 2020 Jan 9]. Available from: https://en.wikipedia.org/wiki/Crime_in_Rivers_State

7. National Population Commission of Nigeria. National Population Commission of Nigeria [Internet]. Census Report. 2017 [cited 2019 Jun 22]. p. 10. Available from: http://www.commonwealthgovernance. org/partners/national-population-commission-of-nigeria/

8. Federal Government of Nigeria. National Bureau of Statistics [Internet]. Vol. X, Population (English Edition). 2006 [cited 2020 Jan 9]. p. 2009-10. Available from: https://nigerianstat.gov.ng/elibrary

9. The Government of Rivers State [Internet]. [cited 2019 Jun 22]. Available from: https://www.riversstate.gov.ng/state

10. Population C. Nigeria Administrative Division (States and Local Government Areas) - Population Statistics, Charts and Map [Internet]. 
2018 [cited 2019 Jun 22]. Available from: http://www.citypopulation. de/php/nigeria-admin.php

11. Kisangau N, Sergon K, Ibrahim Y, et al. Progress towards elimination of measles in Kenya 2003-2016. Pan Afr Med J. 2018; 31: 1-11.

12. Choto R, Chadambuka A, Shambira G, et al. Trends in performance of the National Measles Case-Based Surveillance System, Ministry of Health and Child Welfare Zimbabwe (1999 - 2008). Pan Afr Med J. 2015; 4(1): 5 .

13. Fatiregun AA, Adebowale AS, Fagbamigbe AF. Epidemiology of measles in Southwest Nigeria: An analysis of measles case-based surveillance data from 2007 to 2012. Trans R Soc Trop Med Hyg. 2014; 108(3): 133-40.

14. Khanal S, Sedai TR, Choudary GR, et al. Progress toward measles elimination - Nepal 2007-2014. Morb Mortal Wkly Rep [Internet]. 2016 Mar 4 [cited 2019 Nov 21]; 65(8): 206-10. Available from: http://www.cdc.gov/mmwr/volumes/65/wr/mm6508a3.htm
15. NPC, ICF. Nigeria Demographic and Health Survey 2013. Natl Popul Comm [Internet]. 2014; 566. Available from: http://scholar. google.com/olar?hl=en\&btnG=Search\&q=intitle:Nigeria+Demographic+and+Health+Survey\# $0 \% 5 \mathrm{Cnhttp}: / /$ scholar.google.com/ scholar?hl=en\&btnG=Search\&q=intitle:Nigeria+demographic+and+health+survey+1999\#0

16. Survey H, Indicators K, USAID, UKAID, UNCIEF NB of SON. National Demographic health survey 2018 [Internet]. Vol. 43, First published in the Philippine Journal of Internal Medicine. 2018. Available from: https://dhsprogram.com/pubs/pdf/PR118/PR118.pdf

17. Survey H. National Nutrition and Health Survey (\{NNHeS)\}: First Publ Philipp J Intern Med. 2015; 43(September): 103-15.

18. Strebel PM, Henao-Restrepo AM, Hoekstra E, et al. Global Measles Elimination Efforts: The Significance of Measles Elimination in the United States. J Infect Dis. 2004 May 1; 189(Supplement_1): S251-7. 